\title{
Comparative study on usefulness of modified WHO Partograph in management of labour at one of the rural teaching institutes of Gujarat, India
}

\author{
Shilpa N. Ninama, Mayur R. Gandhi* \\ Department of Obstetrics and Gynecology, GMERS Medical College, Himmatnagrar, Gujarat, India
}

Received: 11 May 2019

Accepted: 17 May 2019

*Correspondence:

Dr. Mayur R. Gandhi,

E-mail: maydeep2008@yahoo.com

Copyright: () the author(s), publisher and licensee Medip Academy. This is an open-access article distributed under the terms of the Creative Commons Attribution Non-Commercial License, which permits unrestricted non-commercial use, distribution, and reproduction in any medium, provided the original work is properly cited.

\begin{abstract}
Background: Modified WHO Partograph is a simple, inexpensive pre-printed form on which labour observation are recorded. It generally comprises three sections of information: maternal condition, fetal condition and labor progress. To study on usefulness of Modified WHO Partograph in management of labour of low risk women, this indirectly improved maternal and perinatal outcome.

Methods: In this study the progress of labour of 150 women with uncomplicated full term pregnancies with cephalic presentation in active labour was studied using modified WHO partograph. 150 historical matched controls comprising of low risk women who delivered without the use of partograph were identified from the labour register and their course of labour studied. The hospital records were studied to obtain the demographic variables. Maternal and perinatal outcome was analyzed for both cases and controls.

Results: The emergency cesarean section rate was reduced from $38.7 \%$ in controls to $24.7 \%$ in cases and both are significant statistically. None of the cases had labour beyond 16 hours, thus indicating significant reduction in prolonged labour. Neonatal intensive care admissions decreased from $18.6 \%$ in controls to $6 \%$ in cases indicating an improved maternal and neonatal outcome.

Conclusions: Modified WHO Partograph work as "early alarming warning system" which help in detecting delayed progress of labour which improves maternal as well as perinatal outcome.
\end{abstract}

Keywords: Cesarean section, Maternal and perinatal outcome, Meconium stained liquor, Modified WHO partograph, Neonatal intensive care unit admission, Prolonge labour

\section{INTRODUCTION}

Pregnancy and child birth is a normal physiological process and every pregnant woman hopes to give birth safely, sadly this is not what is actually happening. Complication can occur anytime during labour. Maternal mortality ratio in India is 130 per 1 , 00,000 live birth. ${ }^{1}$ Maternal death is defined as death of women while pregnant or within 42 days of termination of pregnancy irrespective of duration or site of pregnancy from any cause related to or aggravated by pregnancy or its management but not by accidental or incidental causes (ICD 10). ${ }^{2}$ The most leading cause for maternal morbidity and mortality are severe bleeding (mostly bleeding after childbirth), infections (usually after childbirth), high blood pressure during pregnancy (preeclampsia and eclampsia), complications from delivery and unsafe abortion. ${ }^{3}$

Various methods are used to improve maternal as well as perinatal outcome. Effective management of labour is one of the methods for the same. The partograph (also 
referred to as the partogram) is a simple, inexpensive preprinted form on which labor observations are recorded. It generally comprises three sections of information: maternal condition, fetal condition and labor progress. The form provides a graphic overview of the progression of a woman's labor. Monitoring the progression of labor can assist health care workers in identifying deviations from normal labor progression, first alerting health care workers that a woman may need intervention and then calling for action, if indicated - in the form of direct intervention via labor augmentation or caesarean section, as necessary or referral to a higher-level health facility.

The first graphic assessment of progress of labour was designed by Friedman and further improved by Philpott and Castle. ${ }^{4}$ Much work has been done to improve the partograph as a tool which graphically represents key events during labour and adapts it for use globally. In response to the recommendations of the Safe Motherhood Conference held in Nairobi in 1987, the WHO produced a partograph, and tested its practical value to reduce maternal and perinatal morbidity and mortality. ${ }^{5}$ Partograph use is recommended for routine monitoring of labour, and helps the health care provider in identifying slow progress in labour, and may help initiate appropriate interventions to prevent prolonged and obstructed labour. Very few studies have been used for its use on impact of labour and delivery in the country. Present Study looked for relationship between its use and birth outcomes in teaching hospital.

\section{METHODS}

This study was carried out in a rural medical college of Gujarat where annually 1500 deliveries take place, of which $65 \%$ are low risk population. Each delivery is conducted by a medical officer or junior resident doctor assisted by trained staff nurse who had taken SBA training. The population of the study is all women who seek services at the hospital for labour and delivery. Based on inclusion and exclusion criteria, women admitted in labour room were screened and divided in 2 groups.

\section{Inclusion criteria}

- Patients having complete information of labour on WHO modified partograph

- Low risk uncomplicated full term patients in active labour

- Patients with singleton pregnancy and in cephalic presentation.

\section{Exclusion criteria}

- Patients having incomplete information of labour on WHO modified partograph

- Patients files having information showing high risk factors like severe anaemia, severe hypertension, uncontrolled diabetes, immune compromised status, obstetrical complications like preterm labour, postdatism, antepartum haemorrhage, intrauterine growth restriction and premature rupture of membranes

- Patients with multiple foetus, previous history of cesarean section and with abnormal presentation e.g. breech during labour

Group-1 consider as case group in which 150 uncomplicated full term pregnancies with cephalic presentation in active labour were counted. Women with medical complications like severe anaemia, severe hypertension, uncontrolled diabetes, immune compromised status were excluded from the study. Women with obstetrical complications like preterm labour, postdatism, antepartum haemorrhage, intrauterine growth restriction and premature rupture of membranes were also excluded. Group-2 consider as control group in which women who delivered without the use of partograph were identified from the labour register during the period when partograph was not being used (before 2 years).

The progress of labour of the women in 1st group was plotted on the Modified WHO partograph. Data was collected in a predesigned proforma. Duration of first and second stage of labour and any abnormalities of labour were noted down. Labour parameters like meconium staining of liquor, fetal heart rate abnormalities, need for augmentation of labour, duration of total labour, criteria for intervention if any and need for instrumental delivery or caesarean section were studied. Maternal outcome was analysed by studying various parameters like mode of delivery, postpartum haemorrhage, puerperal sepsis, need for blood transfusion, duration of stay in the hospital and maternal mortality.

The perinatal outcome was analyzed by studying various parameters like condition of baby at birth (live birth/still birth), Apgar score, respiratory distress, neonatal sepsis, need and duration of stay in neonatal intensive care unit. Similar data was collected for the $2^{\text {nd }}$ group. Demographic data and labour delivery data from the record was studied for concluding the above mentioned parameters in the second group.

\section{RESULTS}

Table 1 shows that the mode of delivery was normal in $73.3 \%$ in Group 1(case group) and $57.3 \%$ in Group 2(control group). Rate of caesarean section was higher among control group in which no partograph was used. Both the results were significant statistically.

Table 2 shows indications for caesarean section among both the groups. Foetal distress was the commonest indication for LSCS in both cases and control group. While failure to descent \& prolonged second stage of labour were more common among cases as compared to control group in which partograph was not used. 
Table 1: Distribution according to mode of delivery.

\begin{tabular}{|lllllll|}
\hline Determinants & Group $1($ Cases= 150$)$ & Group $2($ Controls=150) & Z Test & P value \\
\hline Normal delivery & Frequency & Percentages & Frequency & Percentages & & \\
\hline Caesarean delivery & 110 & 73.3 & 86 & 57.3 & 2.9 & 0.003 \\
\hline Instrumental delivery & 37 & 24.7 & 58 & 38.7 & 2.6 & 0.009 \\
\hline
\end{tabular}

Table 2: Distribution according to indication of LSCS.

\begin{tabular}{|lll|}
\hline Indication & Group 1 (Cases=37) & Group 2 (Controls=58) \\
\hline Foetal distress & $20(54.1 \%)$ & $30(51.7 \%)$ \\
\hline Prolonged first stage of labour & $09(24.3 \%)$ & $15(25.9 \%)$ \\
\hline Prolonged second stage of labour & $02(5.4 \%)$ & $05(8.6 \%)$ \\
\hline Cephalo-pelvic disproportion & $02(5.4 \%)$ & $06(10.3 \%)$ \\
\hline Failure to descent & $04(10.8 \%)$ & $02(3.4 \%)$ \\
\hline
\end{tabular}

Table 3: Distribution according to duration of labour.

\begin{tabular}{|lll|}
\hline Duration of labour & Group $1($ Cases=150) & Group 2 (Controls=150) \\
\hline Up to 8 hours & $90(60 \%)$ & $60(40 \%)$ \\
\hline $8-12$ hours & $59(39.3 \%)$ & $43(28.7 \%)$ \\
\hline $12-16$ hours & $1(0.7 \%)$ & $24(16.0 \%)$ \\
\hline$>16$ hours & 00 & $11(7.3 \%)$ \\
\hline Not known & 00 & $12(8.0 \%)$ \\
\hline
\end{tabular}

Table 4: Indication for NICU admissions.

\begin{tabular}{|lll|}
\hline Indications & Group 1 (Cases=10) & Group 2 (Controls=28) \\
\hline Meconium staining of liquor & $5(50 \%)$ & $14(50 \%)$ \\
\hline Respiratory distress & $3(30 \%)$ & $5(17.9 \%)$ \\
\hline Delayed cry & 00 & $05(17.9 \%)$ \\
\hline Low birth weight & $02(20 \%)$ & $03(10.7 \%)$ \\
\hline Altered sensorium of mother & 00 & $01(3.6 \%)$ \\
\hline
\end{tabular}

Table 3 shows that duration of labour was up to 8 hours in $60 \%$ of cases in whom partograph was used as compared to $40 \%$ in control group. Duration was more than 16 hours in $7.3 \%$ of control group where partograph was not applied.

Table 4 shows that in the group where partograph was used it was seen that only $10(6 \%)$ cases require NICU admission as compared to $28(18.6 \%)$ controls where partograph was not used which was significant statistically $(\mathrm{p}<0.05)$. Delayed cry and respiratory distress were found among $35.8 \%$ neonates (17.9\% each) in which partograph were not used.

\section{DISCUSSION}

Present study compared the outcome of pregnancy, maternal and perinatal outcome in the group where partograph was not used Group 2 (Control), to the group in which partograph was used Group 1 (cases). The result of present study reveals that the use of WHO Modified
Partograph improves outcome of labour both in maternal and neonatal point of view.

A significant reduction in the caesarean section rate (38.7\% in control and $24.7 \%$ in cases) was achieved with the introduction of partogram. The finding was similar to the study conducted by Surekha Tayade. ${ }^{6}$ Similar finding of reduction in caesarean section was also recorded in other studies. A caesarean section rate of $0.05 \%$ was achieved by Bird 8 in Papua New Guinea $(0.7 \%$ if symphysiotomies are included). Similar reduction in caesarean section rates was achieved in a study carried out in Pakistan. ${ }^{7}$ In the study by Ahmed B et al, there were $91 \%$ normal vaginal deliveries in partograph group and $81.5 \%$ in other non partograph group. ${ }^{8}$

Considering prolonged labour, it was significantly reduced in the group where partograph was used as compared to other group. Around $7.3 \%$ of control group had prolonged labour of more than 16 hours in present study while none of the case had such prolong labour. 
Javed reported that introduction of partogram showed significant impact on duration of labour $(p<0.001){ }^{7}$ Stduy conducted by Ahmed B et al concluded that the duration of first stage and second stage of labour was significantly decreased in the group where partograph was used. ${ }^{8}$ Similar findings have been reported in other study too. ${ }^{9}$

In present study, rate of NICU admission and its indications were studied in both cases and control. In the group where partograph was used it was seen that only 10 (6\%) cases require NICU admission as compared to 28 $(18.6 \%)$ controls where partograph was not used. Similar finding was noticed in the study by Sethi PS et al. ${ }^{10}$ In present study, it was found that in both the groups Meconium stained Liquor was common indication for NICU admission. Delayed cry as an indication for NICU admission, was seen in $17.9 \%$ of control group where the partograph was not used.

\section{CONCLUSION}

Controlling high rates of maternal mortality is a major challenge in developing countries. Good quality care is very important for reducing maternal mortality. Partograph work as "early alarming warning system" and it can help in detecting delayed progress of labour. It is also useful in decision making for augmentation or termination of labour as well as deciding for surgical intervention. All of these can indirectly lead to improvement in maternal as well as perinatal outcome.

Funding: No funding sources Conflict of interest: None declared

Ethical approval: The study was approved by the Institutional Ethics Committee

\section{REFERENCES}

1. Ayog N. (National Institution for Transforming India), Government of India. Available at: http://niti.gov.in/content/maternal-mortality-ratiommr-100000-live-births.

2. WHO: International classification of diseases and related health problems. Available at: www.who.int/classifications/icd/ICD10 Volume 2_en_2010.pdf.

3. World Health Organization: Maternal Mortality. Available at: https://www.who.int/news-room/factsheets/detail/maternal-mortality.

4. Levin K, KabagemaJd A. Use of the partograph: effectiveness, training, modifications, and barriers-a literature reviews. New York: Engender Health/ Fistula Care; 2011.

5. WHO: World health organization maternal health and safe motherhood programme. Lancet. 1994;343:1399-404.

6. Tayade S, Jadhao P. The impact of use of modified WHO partograph on Maternal and Perinatal outcome. Int J Biomed Adv Res. 2012;03(04):25662.

7. Iffat J, Shereen B, Tabassum S. Role of partogram in preventing prolonged labor. J Pakistan Med Asso. 2007;57(8):408-11.

8. Ahmed B. Partograph versus no partograph: effect on labour progress and delivery outcome: a comparative study. Int J Reprod Contracept Obstet Gynecol. 2017;6(11):4928-33.

9. Windrim R, Seaward PG, Hodnett E, Akoury H. A randomized controlled trial of a bedside partogram in the active management of primiparous labour. J Gynecol Obstet Canada. 2007;29(1):27-34.

10. Sethi PS. Comparative evaluation of composite and simplified who partograms in a tertiary care centre in North India. Int $\mathbf{J}$ Reprod Contracept Obstet Gynecol. 2017;6(11):5003-7.

Cite this article as: Ninama SN, Gandhi MR. Comparative study on usefulness of modified WHO Partograph in management of labour at one of the rural teaching institutes of Gujarat, India. Int J Reprod Contracept Obstet Gynecol 2019;8:2515-8. 